\title{
Studies of the 3D Structure of the Nucleon at JLab
}

Received: date / Accepted: date

\begin{abstract}
Studies of the 3D structure of the nucleon encoded in Transverse Momentum Dependent distribution and fragmentation functions of partons and Generalized Parton Distributions are among the key objectives of the JLab $12 \mathrm{GeV}$ upgrade and the Electron Ion Collider. Main challenges in extracting 3D partonic distributions from precision measurements of hard scattering processes include clear understanding of leading twist QCD fundamentals, higher twist effects, and also correlations of hadron production in target and current fragmentation regions. In this contribution we discuss some ongoing studies and future measurements of spin-orbit correlations at Jefferson Lab.
\end{abstract}

Keywords spin $\cdot$ nucleon structure $\cdot$ parton distributions

\section{Introduction}

The quark-gluon dynamics manifests itself in a set of non-perturbative functions describing all possible spin-spin and spin-orbit correlations, in particular, describing transverse momentum dependent distributions of partons (TMDs), transverse space distributions of partons or Generalized Parton Distributions (GPDs), and Fracture Functions (FFs), defined as conditional probabilities to produce a hadron in the target fragmentation for a given flavor of a struck quark (see Table 1). In recent years, measurements of azimuthal moments of polarized hadronic cross sections in hard processes have emerged as a powerful tool to probe nucleon structure in general and access the TMDs and GPDs, in particular. Many experiments worldwide are currently trying to pin down various effects related to the nucleon structure through semi-inclusive deep-inelastic scattering (HERMES at DESY $[1 ; 2 ; 3 ; 4]$, COMPASS at CERN $[5 ; 6 ; 7 ; 8 ; 9]$, Jefferson Lab $[10 ; 11 ; 12 ; 13 ; 25 ; 14 ; 15])$ polarized proton-proton collisions (PHENIX, STAR and BRAHMS at RHIC) $[16 ; 17 ; 18]$, and electron-positron annihilation (Belle and BaBar) $[19 ; 20 ; 21]$.

Azimuthal distributions of final state particles in semi-inclusive deep inelastic scattering, in particular, are sensitive to the orbital motion of quarks and play an important role in the study of TMDs in the nucleon. The first results on transverse Single Spin Asymmetries (SSAs) in azimuthal distributions of final state hadrons have been reported by the HERMES and COMPASS $[3 ; 5 ; 6 ; 22 ; 8 ; 7 ; 9]$ collaborations, indicating significant azimuthal moments generated both by the Collins [23] and the Sivers [24] effects, describing spin orbit correlations in the final hadronization and initial distribution processes, respectively.

Measurements of SSAs at JLab, performed with longitudinally polarized $\mathrm{NH}_{3}[13]$ and transversely polarized ${ }^{3} \mathrm{He}[25 ; 26 ; 15 ; 14]$, indicate that spin orbit correlations may be significant for certain

H. Avakian

Jefferson Lab

Tel.: +1757-2697764

Fax: +1757-2695800

E-mail: avakian@jlab.org 


\begin{tabular}{|c|c|c|c|c|c|c|c|c|c|c|c|}
\hline $\mathrm{N} / \mathrm{q}$ & $\mathrm{U}$ & $\mathrm{L}$ & $\mathrm{T}$ & & $U$ & $L$ & $T$ & & $U$ & $L$ & $T$ \\
\hline $\mathrm{U}$ & $f_{1}$ & & $h_{1}^{\perp}$ & $U$ & $\mathcal{H}$ & & $\underbrace{\mathcal{E}_{T}}$ & $U$ & $\hat{u}_{1}$ & $\hat{l}_{1}^{\perp h}$ & $\hat{t}_{1}^{h}, \hat{t}_{1}^{\perp}$ \\
\hline $\mathrm{L}$ & & $\mathrm{g}_{1 \mathrm{~L}}$ & $h_{1 L}^{\perp}$ & $L$ & & $\widetilde{\mathcal{H}}$ & $\widetilde{\mathcal{E}}_{T}$ & $L$ & $\hat{u}_{1 L}^{\perp h}$ & $\hat{l}_{1 L}$ & $\hat{t}_{1 L}^{h}, \hat{t}_{1 L}^{\perp}$ \\
\hline $\mathrm{T}$ & $f_{1 T}^{\perp}$ & $g_{1 T}^{\perp}$ & $h_{1}, h_{1 T}^{\perp}$ & $T$ & $\mathcal{E}$ & $\widetilde{\mathcal{E}}$ & $\mathcal{H}_{T}, \widetilde{\mathcal{H}_{T}}$ & $T$ & $\hat{u}_{1 T}^{h}, \hat{u}_{1 T}^{\perp}$ & $\hat{l}_{1 T}^{h}, \hat{l}_{1 T}^{\perp}$ & $\hat{t}_{1 T}, \hat{t}_{1 T}^{h h}, \hat{t}_{1 T}^{\perp h}, \hat{t}_{1 T}^{\perp \perp}$ \\
\hline
\end{tabular}

Table 1 Leading twist TMD distribution functions (left), GPDs (middle) and Fracture Functions (right). The U,L,T correspond to unpolarized, longitudinally polarized, and transversely polarized nucleons (rows) and quarks (columns)
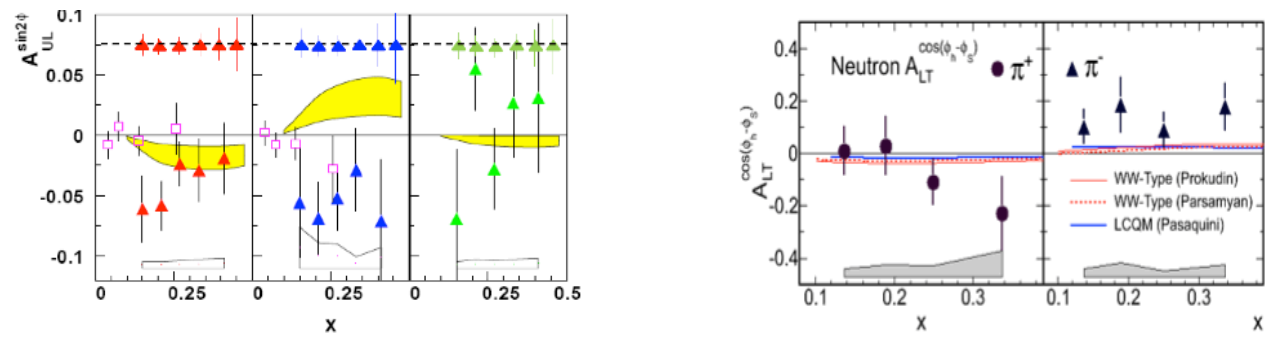

Fig. 1 The Kotzinian-Mulders asymmetry from CLAS (left plot). The open squares and triangles show the existing measurement from HERMES and the preliminary results from CLAS 5.7 GeV CLAS data sets [13], respectively. The curves are calculated using Ref. [27]. The left panel shows the SSA from Hall-A [26].

combinations of spins of quarks and nucleons and transverse momentum of scattered quarks. Single spin asymmetries measured by CLAS with longitudinally polarized protons $\left(\mathrm{NH}_{3}\right.$ target $)$ related to the TMD $h_{1 L}^{\perp}$, describing transversely polarized quarks in the longitudinally polarized nucleon, and the one measured by Hall-A with the transversely polarized neutron (polarized ${ }^{3} \mathrm{He}$ target) related to the TMD $g_{1 T}^{\perp}$, describing longitudinally polarized quarks in the transversely polarized nucleon (see Fig. 1), are consistent with the light-cone quark model predictions [28], as well as lattice calculation [29] for corresponding TMDs, for the expected relative sign of SSAs $A_{U L}^{\sin 2 \phi}$ in $e \pi^{+} X$ and $A_{L T}^{\cos \left(\phi-\phi_{S}\right)}$ in $e \pi^{-} X$.

Significant azimuthal moments in leptoproduction $\left(A_{U U}^{\cos \phi}\right)$, which have been measured in SIDIS already by EMC collaboration [30; 31], were reproduced by latest measurements at CERN, HERMES and JLab $[11 ; 12 ; 32 ; 33 ; 34]$. The first unambiguously measured single spin phenomena in SIDIS, which triggered important theoretical developments, were the sizable longitudinal target $\left(A_{U L}^{\sin \phi}\right)$ and beam $\left(A_{L U}^{\sin \phi}\right)$ spin asymmetries observed at HERMES and JLab $[1 ; 2 ; 10 ; 4]$. They could be interpreted in terms of higher twist distribution functions.

Recent measurements of multiplicities and double spin asymmetries as a function of the final transverse momentum of pions in SIDIS at JLab and HERMES [11; 13; 47] suggest that transverse momentum distributions may depend on the polarization of quarks and possibly also on their flavor. Calculations of transverse momentum dependence of TMDs in different models [35;36;28;37] and on lattice $[29 ; 38]$ indicate that the dependence of the transverse momentum distributions on the quark polarization and flavor may be very significant. Combination of measurements in a large $Q^{2}$ range from HERMES, COMPASS and JLab would allow studies of evolution effects and control possible higher twist contributions in the measurements of TMD observables in general, and of the Sivers asymmetry in particular. Comparison of JLab12 data with HERMES, COMPASS, and future Electron Ion Collider (EIC) (Fig.2) will pin down the non-trivial $Q^{2}$ evolution of Sivers asymmetry.

Much higher $Q^{2}$ range accessible at JLab12 would allow for studies of $Q^{2}$-dependence of different higher twist spin-azimuthal asymmetries (Fig 3), which, apart from providing important information on quark-gluon correlations are needed for understanding of possible corrections from higher twists 

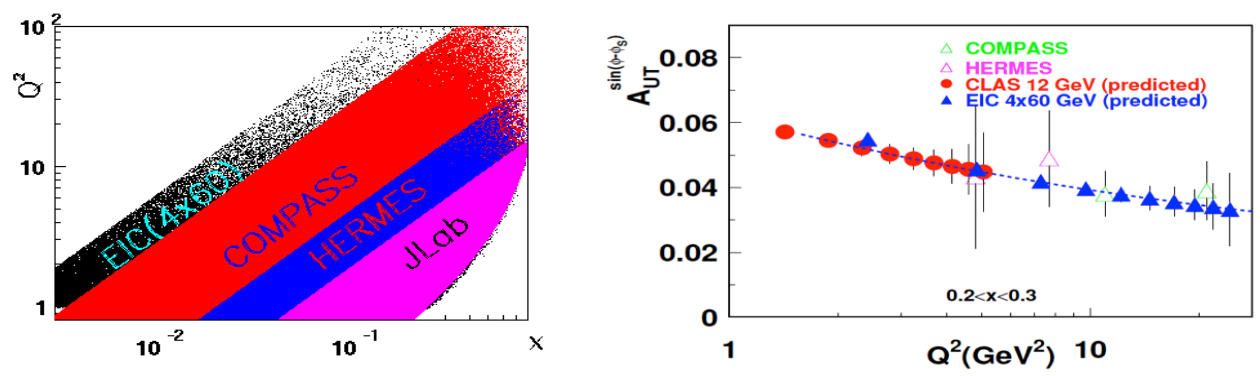

Fig. 2 Comparing CLAS12 and EIC [39] projected data with HERMES [46] and COMPASS [8] results on the Sivers asymmetry.
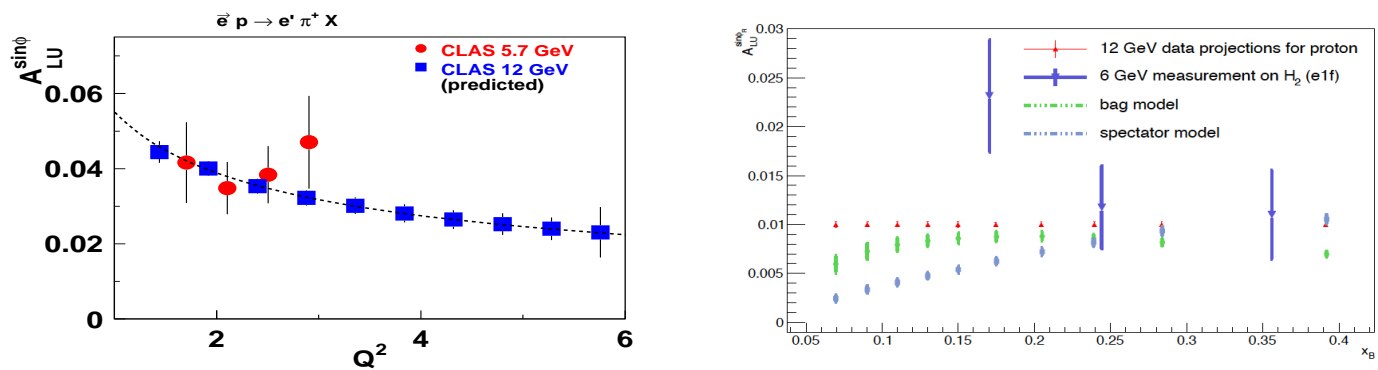

Fig. 3 Projections for higher-twist lepton spin asymmetry $A_{L U}^{\sin \phi}$ for positive pion production as a function of $Q^{2}$ (right) compared to published data from CLAS [10] and projected CLAS12 [40] in one $x, z$ bin $(0.2<x<0.3$, $0.5<z<0.55)$. The right panel shows projections for $x$-dependence of the SSA measurements with CLAS12 using two hadron production [41]. Lines correspond to model predictions based on bag [58] and spectator [42] models.

to leading twist observables. An important process, which can provide independent information on twist-3 TMDs, is the di-hadron production in SIDIS [49]. The projections for corresponding CLAS12 measurements [41] of the SSA in dihadron production as a function of Bjorken- $x$ are shown in Fig.3.

\section{Target fragmentation and back-to-back hadron production}

Although the Target Fragmentation Region (TFR) of DIS, when the hadrons are created from the target remnant, carries important information about the spin and flavor structure of the nucleon, it has not been studied systematically in experiments due to lack of theory fundamentals. The main physical question in the TFR is how the diquark-like remnant system after the DIS process dresses itself up to become a full-fledged hadron, i.e., by which mechanism the quark-antiquark pairs restoring color neutrality are produced, and how this process is correlated with the spin of the target or/and the produced particles. Recently the leading twist formalism for spin and transverse-momentum dependent fracture functions (FFs) has been developed [48]. The structure functions defining the cross section for hadron production in TFR for different polarization states involve unpolarized, longitudinally and transversely polarized quark FFs (see Tab. 1). With hadrons detected in the TFR, the beam SSAs appear already at leading order. With two hadrons detected in the final state, structure functions may depend also on the relative azimuthal angle of the two hadrons, generating a long range correlation between hadrons produced in CFR and TFR. The kinematic plane for back-to-back hadron production is shown on Fig. 4. 

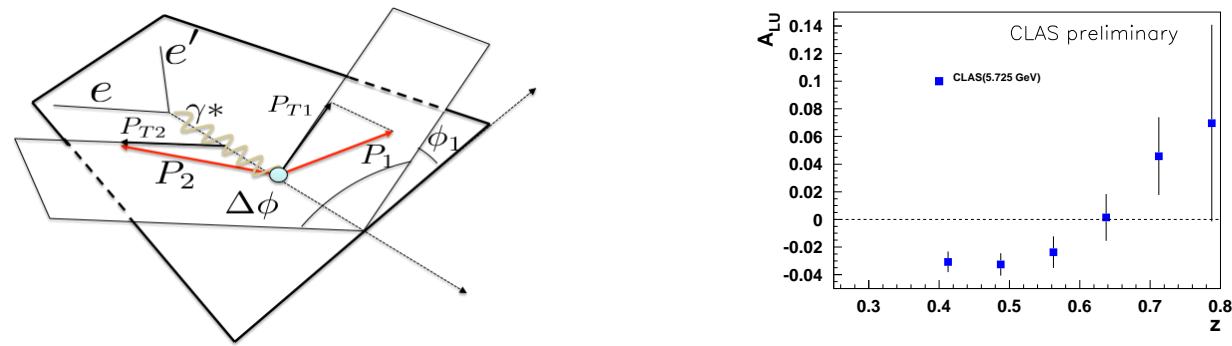

Fig. 4 Kinematic plane for back-to-back (b2b) hadron production in SIDIS (left) and measured SSA in backto-back proton and $\pi^{+}$production as a function of the product of their transverse momenta $P_{T 1} P_{T 2}$.

Choosing as independent azimuthal angles $\Delta \phi=\phi_{2}-\phi_{1}$ and $\phi_{2}$, the beam spin asymmetry could be defined as

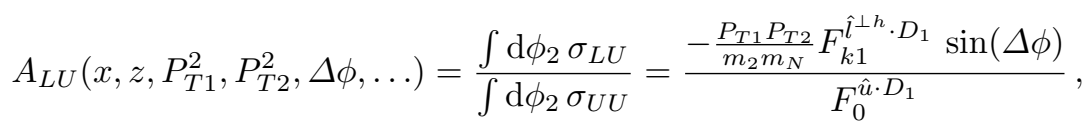

where $z$ is the fraction of the virtual photon energy carried by the forward pion, $P_{T 1}, P_{T 2}$ are transverse momenta of the final state hadrons, and structure functions $F_{0}^{\hat{u} \cdot D_{1}}$ and $F_{k 1}^{\hat{l}^{\perp h} \cdot D_{1}}$ are convolutions of unpolarized and polarized FFs with the standard unpolarized fragmentation function $D_{1}$. First measurements of single spin asymmetry defined by Eq.1 in semi-inclusive production of protons and charged pions in coincidence with the scattered electron in hard scattering kinematics $\left(Q^{2}>1 \mathrm{GeV}^{2}\right.$, $W^{2}>4 \mathrm{GeV}^{2}$ ) have been performed by the CLAS collaboration using $5.5 \mathrm{GeV}$ and $5.7 \mathrm{GeV}$ longitudinally polarized electron beams scattering off a 5-cm-long liquid-hydrogen target (CLAS e1f and e16 experiments). The beam spin asymmetry, $A_{L U}^{\sin \Delta \phi}$, has been calculated as a sinusoidal modulation of the difference of azimuthal angles of proton and $\pi^{+}$with respect to the lepton scattering plane, for different electron helicity states. The modulation was extracted for different bins in $x, z$ of the pion and the product of transverse momenta of final state proton and pion with respect to the virtual photon in the CM frame. Target and current fragmentation regions were selected by cuts on the $x_{F}$ variables of protons $\left(x_{F}<0\right)$ and pions $\left(x_{F}>0\right)$.

Significant asymmetries in b2b production of protons and pions have been observed for all 3 pions in the final state. The $z$-dependence of the SSA shows a trend for asymmetry (see Fig.4) to change the sign at large $z$, indicating opposite sign in the exclusive limit. With a cut on the missing mass of the el $\pi^{+} X$ system, $M_{X}>1.4$ we have almost no data at $z>0.7$.

The $x$-dependence of the the $A_{L U}^{\sin \phi}$ is consistent with asymmetry being large in the large- $x$ region, were the valence quark presence is very significant. Similar behavior has been observed also for events with neutral and negative pions in the final state, detected in coincidence with scattered electron and proton in the target fragmentation region.

\section{Hard Exclusive Processes and GPDs}

Measurements of various observables in hard exclusive production versus the momentum transfer to the nucleon, $t$, on other hand, provide the information necessary for transverse nucleon imaging [50]. The different nucleon spin components of the GPDs [44; 43] describing unpolarized and longitudinally polarized quarks (Table 1) can be extracted by measuring spin asymmetries in Deeply Virtual Compton Scattering (DVCS) with polarized targets. Information about the flavor decomposition requires measurements with both protons and neutrons. The latest measurements of DVCS cross sections from unpolarized proton target from JLab Halls A\&B [59; 52] are consistent with theory predictions for a rapid change of the cross section with azimuthal angle, due to Bethe-Heitler propagators, pushing the DVCS to smaller bins in the azimuthal angle $\phi$ for separation of different azimuthal moments. Further separation of contributions from unpolarized GPDs, and in particular polarized GPDs, require measurements with longitudinally and transversely polarized targets. Latest measurements of target spin 

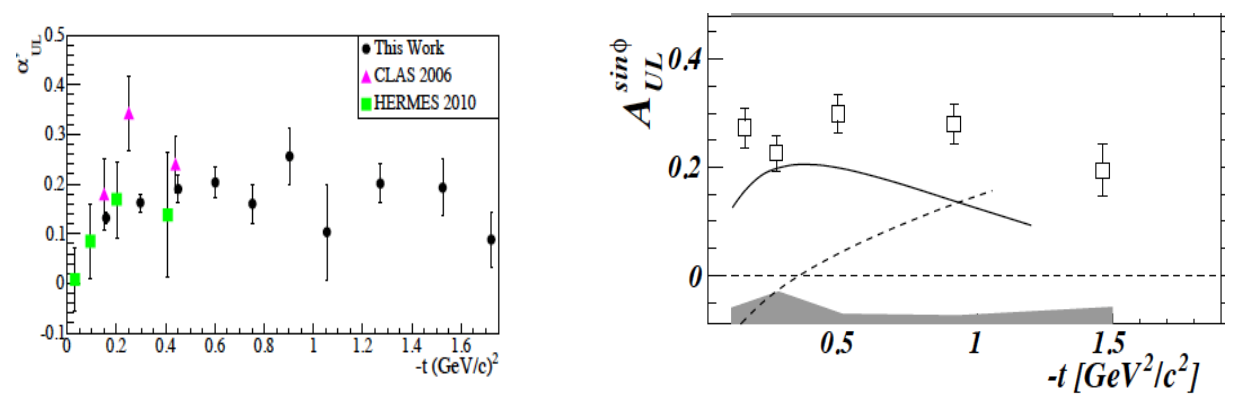

Fig. 5 Spin asymmetries using the Hall-B polarized $\mathrm{NH}_{3}$ target for exclusive photons [61] and neutral pions [60] for $\left\langle Q^{2}\right\rangle=1.94(\mathrm{GeV} / \mathrm{c})^{2},\langle x\rangle=0.25$

asymmetries in DVCS from CLAS collaboration (see Fig. 5) suggests that $t$-dependence of polarized quark GPDs may be significantly different than for the unpolarized GPDs, indicating that transverse space distributions of quarks may depend on their spin.

Additional information about the spin/flavor separation can come from meson production data. In particular pseudoscalar meson electroproduction provides access to transversely polarized quark GPDs, $H_{T}^{q}, E_{T}^{q}, \tilde{H}_{T}^{q}, \tilde{E}_{T}^{q}$, corresponding to parton helicity-flip (chiral-odd) processes [53], not accessible in DVCS studies [56]. For example, the chiral-odd GPD $H_{T}$ encode unique information on the transverse spin structure of the proton and on the tensor charge since it is defined as a generalization of the quarks transversity distribution, $h_{1}(x)$. Exclusive pseudoscalar meson electroproduction provides a unique possibility to access the "transversity" or chiral-odd GPDs through polarized scattering measurements [45]. Other important observables accessible in hard exclusive production of pseudoscalar mesons include the GPD $\bar{E}_{T}$ describing the transverse space distributions of transversely polarized quarks in the unpolarized nucleon and the longitudinal-transverse (LT) correlation provided by $\widetilde{E}_{T}$, which may be relevant for interpretation of orbital angular momentum [51].

Measurements of different final state mesons, $\pi^{o}, \eta$ and $K$ will provide important complementary information for flavor decomposition of the various GPDs [55; 54], and in particular, help to further improve the precision of the tensor charge. The measurements for $\pi^{o}$ and $\eta$ SSAs over a large kinematic range are challenging, since their cross sections are small (compared to charged pions). In addition the clean detection of $\pi^{\circ} \mathrm{s}$ and $\eta \mathrm{s}$ requires the measurement of their two decay photons. Since the precision attainable in charge particle detection is typically much higher, charged kaons provide a very attractive alternative to neutral mesons channels in studies of transversity GPDs. It has been argued that the $H_{T}$ effects are essential in the $K^{+} \Lambda$ channel while in the $K^{+} \Sigma^{0}$ leptoproduction the $\bar{E}_{T}$ contribution is mostly important [57]. Predictions for the cross section of the $K^{+} \Lambda$ and $K^{+} \Sigma^{0}$ leptoproduction [57] has been used to justify future measurements with CLAS12 [62].

The future measurements with the $12 \mathrm{GeV}$ upgrade of Jefferson Lab will be essential for the study of the transverse momentum and transverse space dependent distributions in the valence region. At JLab there are several closely-related proposals approved to measure spin and azimuthal asymmetries in all three Halls, providing complementary studies of different aspects of the complex structure of the nucleon in terms of flavor, momentum and spin. 


\section{References}

1. Airapetian A, et al (2000) Observation of a single-spin azimuthal asymmetry in semi- inclusive pion electroproduction. Phys Rev Lett 84:4047-4051, hep-ex/9910062

2. Airapetian A, et al (2001) Single-spin azimuthal asymmetries in electroproduction of neutral pions in semi-inclusive deep-inelastic scattering. Phys Rev D64:097,101, hep-ex/0104005

3. Airapetian A, et al (2005) Single-spin asymmetries in semi-inclusive deep-inelastic scattering on a transversely polarized hydrogen target. Phys Rev Lett 94:012,002, hep-ex/0408013

4. Airapetian A, et al (2007) Beam-spin asymmetries in the azimuthal distribution of pion electroproduction. Phys Lett B648:164-170, hep-ex/0612059

5. Alexakhin VY, et al (2005) First measurement of the transverse spin asymmetries of the deuteron in semi-inclusive deep inelastic scattering. Phys Rev Lett 94:202,002, hep-ex/0503002

6. Ageev ES, et al (2007) A new measurement of the collins and sivers asymmetries on a transversely polarised deuteron target. Nucl Phys B765:31-70, hep-ex/0610068

7. Alekseev MG, et al (2010) Azimuthal asymmetries of charged hadrons produced by high energy muons scattered off longitudinally polarised deuterons. Eur Phys J C70:39-49, DOI 10.1140/epjc/s10052-0101461-9, 1007.1562

8. Alekseev MG, et al (2010) Measurement of the Collins and Sivers asymmetries on transversely polarised protons. Phys Lett B692:240-246, DOI 10.1016/j.physletb.2010.08.001, 1005.5609

9. Adolph C, et al (2012) Transverse spin effects in hadron-pair production from semi-inclusive deep inelastic scattering. PhysLett B713:10-16, DOI 10.1016/j.physletb.2012.05.015, 1202.6150

10. Avakian H, et al (2004) Measurement of beam-spin asymmetries for deep inelastic pi+ electroproduction. Phys Rev D69:112,004, hep-ex/0301005

11. Mkrtchyan H, et al (2008) Transverse momentum dependence of semi-inclusive pion production. Phys Lett B665:20-25, hep-ph/0709.3020

12. Osipenko M, et al (2009) Measurement of unpolarized semi-inclusive pi+ electroproduction off the proton. Phys Rev D80:032,004, hep-ex/0809.1153

13. Avakian H, et al (2010) Measurement of Single and Double Spin Asymmetries in Deep Inelastic Pion Electroproduction with a Longitudinally Polarized Target. Phys Rev Lett 105:262,002, DOI 10.1103/PhysRevLett.105.262002, hep-ex/1003.4549

14. Zhang Y, et al (2014) Measurement of pretzelosity asymmetry of charged pion production in SemiInclusive Deep Inelastic Scattering on a polarized ${ }^{3} \mathrm{He}$ target. Phys Rev C90(5):055,209, DOI 10.1103/PhysRevC.90.055209, 1312.3047

15. Zhao YX, et al (2014) Single spin asymmetries in charged kaon production from semi-inclusive deep inelastic scattering on a transversely polarized ${ }^{3} \mathrm{He}$ target. Phys Rev C90(5):055,201, DOI 10.1103/PhysRevC.90.055201, 1404.7204

16. Adams J, et al (2004) Cross sections and transverse single-spin asymmetries in forward neutral pion production from proton collisions at $\mathrm{s}^{* *}(1 / 2)=200-\mathrm{GeV}$. Phys Rev Lett 92:171,801, hep-ex/0310058

17. Chiu M (2007) Single spin transverse asymmetries of neutral pions at forward rapidities in $\mathrm{s}^{* *}(1 / 2)=$ 62.4-GeV polarized proton collisions in PHENIX. AIP Conf Proc 915:539-542, nucl-ex/0701031

18. Arsene I, et al (2008) Single Transverse Spin Asymmetries of Identified Charged Hadrons in Polarized p+p Collisions at $\sqrt{s}=62.4 \mathrm{GeV}$. Phys Rev Lett 101:042,001, nucl-ex/0801.1078

19. Abe K, et al (2006) Measurement of azimuthal asymmetries in inclusive production of hadron pairs in e+ e- annihilation at Belle. Phys Rev Lett 96:232,002, hep-ex/0507063

20. Vossen A, et al (2011) Observation of the interference fragmentation function for charged pion pairs in $e^{+} e^{-}$annihilation near $\sqrt{s}=10.58 \mathrm{GeV}$. PhysRevLett 1104.2425

21. Lees JP, et al (2014) Measurement of Collins asymmetries in inclusive production of charged pion pairs in $e^{+} e^{-}$annihilation at BABAR. Phys Rev D90(5):052,003, DOI 10.1103/PhysRevD.90.052003, 1309.5278

22. Airapetian A, et al (2010) Effects of transversity in deep-inelastic scattering by polarized protons. Phys Lett B693:11-16, DOI 10.1016/j.physletb.2010.08.012, 1006.4221

23. Collins JC (1993) Fragmentation of transversely polarized quarks probed in transverse momentum distributions. Nucl Phys B396:161-182, hep-ph/9208213

24. Sivers DW (1991) Hard scattering scaling laws for single spin production asymmetries. Phys Rev D43:261263

25. Qian X, et al (2011) Single Spin Asymmetries in Charged Pion Production from Semi-Inclusive Deep Inelastic Scattering on a Transversely Polarized ${ }^{3}$ He Target. Phys Rev Lett 107:072,003, DOI 10.1103/PhysRevLett.107.072003, 1106.0363

26. Huang J, et al (2012) Beam-Target Double Spin Asymmetry A_LT in Charged Pion Production from Deep Inelastic Scattering on a Transversely Polarized He-3 Target at $1.4<Q^{2}<2.7 \mathrm{GeV}^{2}$. Phys Rev Lett 108:052,001, DOI 10.1103/PhysRevLett.108.052001, 1108.0489

27. Efremov AV, Goeke K, Schweitzer P (2005) Transversity distribution in spin asymmetries in semi- inclusive dis and in the drell-yan process. Czech J Phys 55:A189-A208, hep-ph/0412420

28. Pasquini B, Cazzaniga S, Boffi S (2008) Transverse momentum dependent parton distributions in a lightcone quark model. Phys Rev D78:034,025, hep-ph/0806.2298

29. Hagler P, Musch BU, Negele JW, Schafer A (2009) Intrinsic quark transverse momentum in the nucleon from lattice QCD. Europhys Lett 88:61,001, hep-lat/0908.1283

30. Aubert JJ, et al (1983) Measurement of hadronic azimuthal distributions in deep inelastic muon proton scattering. Phys Lett B130:118 
31. Arneodo M, et al (1987) Measurement of hadron azimuthal distributions in deep inelastic muon proton scattering. Z Phys C34:277

32. Kafer W (2008) Measurements of Unpolarized Azimuthal Asymmetries at COMPASS. Transversity 2008 proceedings hep-ex/0808.0114

33. Giordano F, Lamb R (2009) Measurement of azimuthal asymmetries of the unpolarized cross section at HERMES. AIP Conf Proc 1149:423-426, hep-ex/0901.2438

34. Gohn W, Avakian H, Joo K, Ungaro M (2009) Beam spin asymmetries from semi-inclusive pion electroproduction in deep inelastic scattering. AIP Conf Proc 1149:461-464, DOI 10.1063/1.3215689

35. Lu Z, Ma BQ (2004) Sivers function in light-cone quark model and azimuthal spin asymmetries in pion electroproduction. Nucl Phys A741:200-214, DOI 10.1016/j.nuclphysa.2004.06.006, hep-ph/0406171

36. Anselmino M, Efremov A, Kotzinian A, Parsamyan B (2006) Transverse momentum dependence of the quark helicity distributions and the cahn effect in double-spin asymmetry a(ll) in semi inclusive dis. Phys Rev D74:074,015, hep-ph/0608048

37. Bourrely C, Buccella F, Soffer J (2011) Semiinclusive DIS cross sections and spin asymmetries in the quantum statistical parton distributions approach. PhysRev D83:074,008, DOI 10.1103/PhysRevD.83.074008, 1008.5322

38. Musch BU, Hagler P, Negele JW, Schafer A (2011) Exploring quark transverse momentum distributions with lattice QCD. PhysRev D83:094,507, DOI 10.1103/PhysRevD.83.094507, 1011.1213

39. Accardi A, Albacete J, Anselmino M, Armesto N, Aschenauer E, et al (2012) Electron Ion Collider: The Next QCD Frontier - Understanding the glue that binds us all 1212.1701

40. Avakian H, et al (2008) Transverse spin effects in sidis at 12 gev with transversely polarized target. JLab Experiment E12-06-015

41. Pisano S, et al (2014) Higher-twist collinear structure of the nucleon through di-hadron sidis on unpolarized hydrogen and deuterium. JLab Experiment E12-06-112B/E12-09-008B http://www.jlab.org/exp_prog/proposals/14/E12-06-112B_E12-09-008B.pdf

42. Jakob R, Mulders PJ, Rodrigues J (1997) Modelling quark distribution and fragmentation functions. Nucl Phys A626:937-965, hep-ph/9704335

43. Ji XD (1997) Deeply-virtual compton scattering. Phys Rev D55:7114-7125, hep-ph/9609381

44. Radyushkin AV (1996) Scaling limit of deeply virtual compton scattering. Phys Lett B380:417-425, hep$\mathrm{ph} / 9604317$

45. Ahmad S, Goldstein GR, Liuti S (2009) Nucleon Tensor Charge from Exclusive $\pi^{\circ}$ Electroproduction. Phys Rev D79:054,014, DOI 10.1103/PhysRevD.79.054014, 0805.3568

46. Airapetian A, et al (2009) Observation of the Naive-T-odd Sivers Effect in Deep Inelastic Scattering. Phys Rev Lett 103:152,002, DOI 10.1103/PhysRevLett.103.152002, 0906.3918

47. Airapetian A, et al (2013) Multiplicities of charged pions and kaons from semi-inclusive deep-inelastic scattering by the proton and the deuteron. PhysRev D87:074,029, DOI 10.1103/PhysRevD.87.074029, 1212.5407

48. Anselmino M, Barone V, Kotzinian A (2011) SIDIS in the target fragmentation region: polarized and transverse momentum dependent fracture functions. Phys Lett B699:108-118, DOI 10.1016/j.physletb.2011.03.067, 1102.4214

49. Bacchetta A, Radici M (2004) Two-hadron semi-inclusive production including subleading twist. Phys Rev D69:074,026, DOI 10.1103/PhysRevD.69.074026, hep-ph/0311173

50. Burkardt M (2003) Impact parameter space interpretation for generalized parton distributions. Int J Mod Phys A18:173-208, DOI 10.1142/S0217751X03012370, hep-ph/0207047

51. Courtoy A, Goldstein GR, Hernandez JOG, Liuti S, Rajan A (2014) On the Observability of the Quark Orbital Angular Momentum Distribution. Phys Lett B731:141-147, DOI 10.1016/j.physletb.2014.02.017, 1310.5157

52. Defurne M, et al (2015) E00-110 experiment at Jefferson Lab Hall A: Deeply virtual Compton scattering off the proton at $6 \mathrm{GeV}$. Phys Rev C92(5):055,202, DOI 10.1103/PhysRevC.92.055202, 1504.05453

53. Diehl M (2003) Generalized parton distributions. Phys Rept 388:41-277, DOI 10.1016/j.physrep.2003.08.002, hep-ph/0307382

54. Frankfurt L, Pobylitsa P, Polyakov MV, Strikman M (1999) Hard exclusive pseudoscalar meson electroproduction and spin structure of a nucleon. PhysRev D60:014,010, DOI 10.1103/PhysRevD.60.014010, hep-ph/9901429

55. Goeke K, Polyakov MV, Vanderhaeghen M (2001) Hard Exclusive Reactions and the Structure of Hadrons. Prog Part Nucl Phys 47:401-515, DOI 10.1016/S0146-6410(01)00158-2

56. Goloskokov SV, Kroll P (2010) An attempt to understand exclusive pi+ electroproduction. Eur Phys J C65:137-151, DOI 10.1140/epjc/s10052-009-1178-9, 0906.0460

57. Goloskokov SV, Kroll P (2011) Transversity in hard exclusive electroproduction of pseudoscalar mesons Eur Phys J A47:112, DOI 10.1140/epja/i2011-11112-6, 1106.4897

58. Jaffe RL, Ji X (1992) Chiral odd parton distributions and drell-yan processes. Nucl Phys B375:527-560

59. Jo HS, et al (2015) Cross sections for the exclusive photon electroproduction on the proton and Generalized Parton Distributions. Phys Rev Lett 115(21):212,003, DOI 10.1103/PhysRevLett.115.212003, 1504.02009

60. Kim A, et al (2015) Target and Double Spin Asymmetries of Deeply Virtual $\pi^{0}$ Production with a Longitudinally Polarized Proton Target and CLAS. Submitted to PRL 1511.03338

61. Pisano S, et al (2015) Single and double spin asymmetries for deeply virtual Compton scattering measured with CLAS and a longitudinally polarized proton target. Phys Rev D91(5):052,014, DOI 10.1103/PhysRevD.91.052014, 1501.07052

62. Kim A, et al (2014) Studies of chiral-odd gpds using the clas12 detector. LOI-12-14-003 